\title{
DEVIATION OF THE AORTIC SEPTUM: COMPLETE TRANSPOSITION OF THE GREAT VESSELS, WITH REPORT OF TWO CASES \\ IN INFANTS *
}

\author{
VICTOR C. JACOBSON, M.D. \\ Resident Pathologist of the Peter Bent Brigham Hospital \\ BOSTON
}

Congenital cardiac abnormalities are encountered sufficiently often to emphasize the manifold possibilities of such defective development, their frequent multiplicity of expression in a single case, and the difficulties in the way of recognizing antemortem the actual lesions present. Such signs as cyanosis since birth, a loud murmur and thrill at the base of the heart, and clubbed fingers-when taken together are quite pathognomonic of congenital heart disease. But defects of the organ acquired in utero are seldom single, and the mechanical explanation of the physical findings is often a difficult problem.

Complete transposition of the great vessels is uncommon enough to warrant publication of such cases as are proved at necropsy. I have found two instances of this condition in infants, one living three weeks and the other twelve months, the duration of life and the relation of the heart lesions thereto furnishing an instructive feature. The cases are from the clinic of Dr. Chas. Hunter Dunn at the Infant's Hospital, Boston, and I am indebted to him for the clinical data.

\section{REPORT OF CASES}

CAse 1.-Male, white, aged 12 months.

Family History.-Father and mother are living and well. There is no history of miscarriages, tuberculosis or syphilis.

Past History.-Born at full term, normal delivery, weighing $5 \frac{1}{2}$ pounds. Cried normally but was blue. He has had an occasional cold.

Present Illness.-Baby has been blue since birth, especially when he cries. $\mathrm{He}$ is sent in with a diagnosis of congenital heart disease and for regulation of his feeding.

Physical Examination. - Baby is cyanotic, more so on crying. Anterior fontanel admits two fingers. Craniotabes present. There is right interscapular dulness and spinal dulness to the level of the fourth vertebra. Heart: There is a palpable thrill and diffuse impulse over the precordium. Enlargement is made out $2 \mathrm{~cm}$. outside the left nipple line, and $3 \mathrm{~cm}$. from the midsternal line to the right. A systolic murmur is heard at the apex and faintly in the vessels of the neck

A week later the following note was made: "There is still marked cyanosis which is increased by crying. The cardiac impulse is diffuse and there is a

* Received for publication Sept. 23, 1920. 
palpable thrill. The heart borders are unchanged. A loud systolic murmur is heard all over the precordium, transmitted into the left axilla and back but not into the vessels of the neck."

Diagnosis.-Pulmonic stenosis and patent interventricular septum.

Pulse rate varied between 135 and 170 per minute and respirations from 37 to 78. The following week he developed a fever and chest signs of bronchopneumonia and died.

Vecropsy Diagnoses.-Complete transposition of aorta and pulmonary artery; patent foramen ovale; patent interventricular septum; vegetative endocarditis of the wall of the septum defect; vegetative endocarditis of the tricuspid valve with insufficiency; right auricular and ventricular hypertrophy; passive congestion of the lungs.

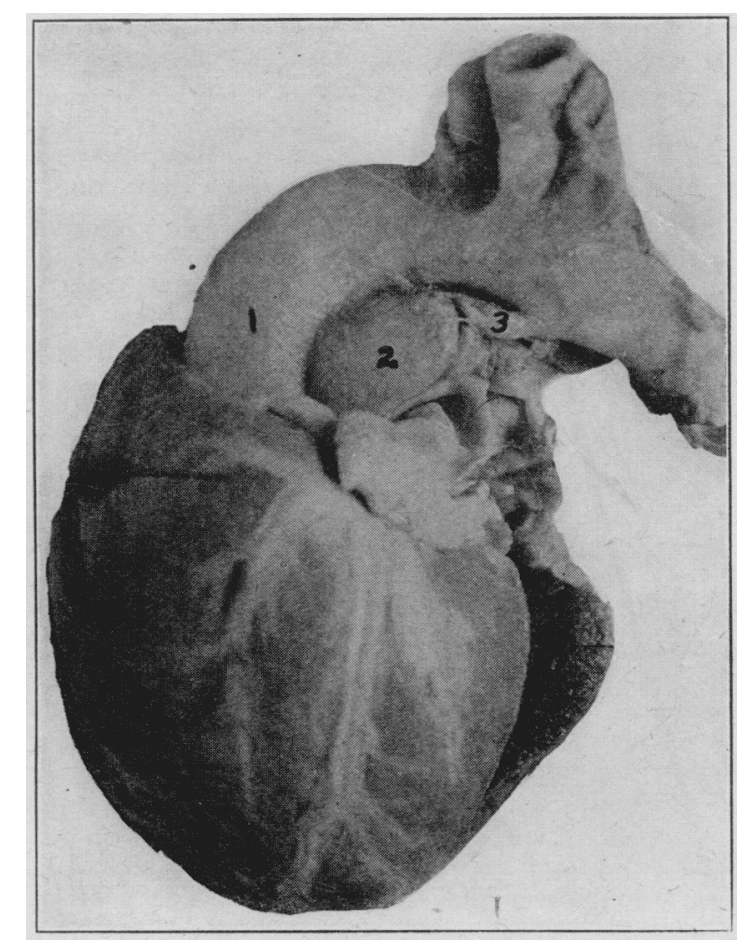

Fig. 1.-Case 1. Heart, anterior aspect. 1. Aorta. 2. Pulmonary artery. 3. Ductus arteriosus.

Body is that of a somewhat emaciated male white infant, $60 \mathrm{~cm}$. in length. There is slight postmortem lividity. The primary teeth are present. There are no external stigmata and no clubbing of the fingers.

Peritoneal Cavity: The abdominal viscera are in normal positions. The urachus is closed.

Pleural Cavity: Contains no free fluid.

Mediastinum: The thymus is small and of loose texture, weighing about $4 \mathrm{gm}$. The greater part of the mediastinum is occupied by the enlarged heart which lies in about normal position.

Pericardial Cavity: Contains about 10 c.c. of clear fluid.

Heart: Weight, $76 \mathrm{gm}$; diameters, 8.5 by $7 \mathrm{~cm}$. Several abnormalities are apparent on opening the pericardium. It is considerably enlarged, the right 
auricle markedly dilated and placed rather posteriorly. The general contour of the organ follows about normal lines, although the anteroposterior diameter is probably increased out of proportion to the general hypertrophy. Viewed anteriorly, the most conspicuous anomaly is the origin of the aorta, this vessel arising from the conus arteriosus of the right ventricle a little to the right of the usual origin of the pulmonary artery.

The pulmonary artery has its origin in the left ventricle, whence it arises to the left of and slightly posteriorly to the aorta, the greater part of the arch of which is in close apposition to the convexity of the pulmonary artery. The left auricle is small and empty, but its appendage is rather elongated, extending about $0.5 \mathrm{~cm}$. along the anterior longitudinal sulcus.

The superior and inferior venae cavae enter the dilated right auricle, their ostia being $1 \mathrm{~cm}$. apart, and that of the inferior vessel being high up on the posterior wall.

The aorta is $1.3 \mathrm{~cm}$. in diameter at its base. It gives off two main coronary branches, the right and left, the left making its exit in plain view anterior to the pulmonary artery, passing under the left auricular appendage where it gives off its auricular and anterior circumflex branches. From the aorta arise in a normal manner the innominate, left common carotid and left subclavian arteries. The ductus arteriosus is 1 by $0.3 \mathrm{~cm}$. and connects the pulmonary artery at a point on the upper anterior wall just proximal to its primary division, to the aorta just below the origin of the left subclavian artery. It is entirely closed by an endarteritis.

The incision through the tricuspid valve and lateral wall of the right ventricle encounters a greatly hypertrophied myocardium. That of the right auricle is hypertrophied in proportion. The foramen ovale is widely open, the patency being $1.2 \mathrm{~cm}$. in diameter, practically round in outline and bordered by a rather high limbus. The tricuspid valve leaflets are nodular and thickened, pale ragged vegetations covering the free borders and producing sufficient puckering to cause a high degree of insufficiency. The circumference of the valve orifice is $4.7 \mathrm{~cm}$. The chordae tendineae are taut and the papillary muscles greatly hypertrophied.

In the membranous portion of the interventricular septum is a defect $0.8 \mathrm{~cm}$. wide and rounded in outline. The greater part is occluded by a mass of cauliflower-like granulations so that an actual opening of only $0.8 \mathrm{~cm}$. exists along the lower border.

The left auricle is small and its wall is only $0.1 \mathrm{~cm}$. thick (after fixation). The mitral valve is normal. The left ventricle wall is only $0.4 \mathrm{~cm}$. thick. From the left ventricle $1 \mathrm{~cm}$. above and to the left of the interventricular septum defect the pulmonary artery arises. It gives off in an essentially normal manner the right and left pulmonary branches. The position of the ductus arteriosus has been described. The main pulmonary artery is $0.8 \mathrm{~cm}$. in diameter just above its valve and there is no evidence of stenosis.

5 The lesions in this heart consist, therefore, of complete transposition of aorta and pulmonary artery, with reversal of the usual muscular preponderance of the two ventricles. The right auricle is markedly dilated and hypertrophied. The large patent foramen ovale makes virtually a single chamber of the auricles. The interventricular septum defect is practically closed by granulations so that the minute opening still present is probably of little consequence.

Lungs: Weight of right, $52 \mathrm{gm}$.; left, $38 \mathrm{gm}$. The posterior two-thirds of each lung are similar in appearance, being dark red on the pleural surface and boggy. The anterior portions are pink and crepitant. On section, from the anterior portions exudes a pale frothy fluid and from the posterior a bloody, frothy fluid. The alveolar outlines are as a whole quite distinct. The trachea and primary bronchi are normal.

Spleen: Weight, $14 \mathrm{gm}$. It presents the appearance of chronic passive congestion. 
Gastro-Intestinal Tract: Appears normal

Pancreas: Appears normal.

Liver: Weight, $186 \mathrm{gm}$. It is dark red with a smooth capsular surface. On section much dark red blood escapes from the hepatic veins. The lobules are indistinctly outlined by pale red lines.

Gallbladder, Kidneys, Suprarenals and Bladder: Appear normal.

Aorta: Shows no abnormality in its branching.

CASE 2,-Male, white, aged 3 weeks.

Family History. - Father and mother well. There is no history of miscarriages.

Past History:- $-\mathrm{He}$ is the second baby and of normal birth. There have been no convulsions nor disturbance of feeding. The baby is brought to the hospital because of marked cyanosis and with the diagnosis of a "bad heart."

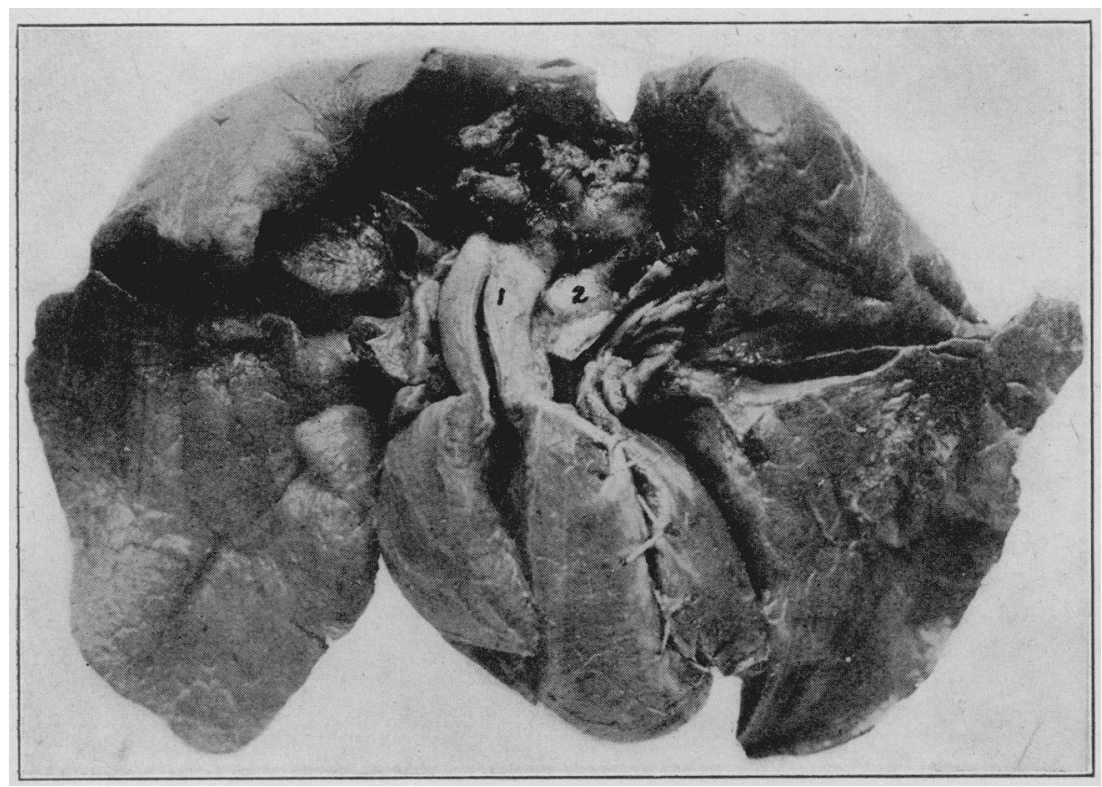

Fig. 2.-Case 2. Heart and lungs, anterior aspect. 1. Aorta. 2. Pulmonary artery. 3. Ductus arteriosus.

Physical Examination,-Baby has marked cyanosis, more especially over the face and becoming worse with crying. There is moderate dyspnea. The lungs are fairly clear throughout. Over the heart a systolic murmur is heard at the base at the junction of the second rib and sternum on the left.

Diagnosis.-Pulmonic stenosis (?). Malformation of the vessels. RoentgenRay Report: Slight enlargement of the heart to the left. The right hilus is suggestive of atelectasis. Pulse rate varied between 140 and 150 per minute: respirations from 40 to 60 . Death occurred twenty-three days after entrance.

Necropsy Diagnoses.-Complete transposition of aorta and pulmonary artery; patent ductus arteriosus; slightly patent foramen ovale; congestion of thoracic and abdominal viscera: mongolian facies; accessory spleens.

Body is that of a fairly well nourished male, white infant, $50 \mathrm{~cm}$. in length. There are no external stigmata except for slight mongolian aspect of the eyes and some outward bowing of the legs with slightly enlarged epiphyses. The anterior fontanel is open $2 \mathrm{~cm}$. and somewhat depressed. 
Peritoneal Cavity: There are no adhesions or free fluid. The organs are in normal positions. The diaphragm on both sides is level with the fourth rib.

Pleural and Pericardial Cavities: Contain no excess of fluid.

Heart: As it lies in situ it appears rather larger than normal in every respect. The left side is soft and collapsed, altering the usual rounded outline of this part of the organ. The ascending aorta is prominent, completely covering from view the pulmonary artery and externally apparently coming off from the right side of the heart. The heart, empty, measures 4.8 by $4.2 \mathrm{~cm}$. There is marked hypertrophy of the myocardium of the right ventricle, the wall being $1.7 \mathrm{~cm}$. in thickness, the left ventricle $0.3 \mathrm{~cm}$. The valves are smooth and glistening and there are no signs of stenosis. The ventricles contain a scant amount of fluid blood. The auricular appendages are empty. There is complete transposition of the great vessels, the aorta coming from the right ventricle immediately to the right of the interventricular septum and the pulmonary artery from the left ventricle, about $0.5 \mathrm{~cm}$. to the left of the septum. The superior and inferior venae cavae enter the right auricle and the pulmonary veins enter the left auricle in a normal manner. There is a patulous ductus arteriosus which measures $1.5 \mathrm{~cm}$. in length with a lumen of $3 \mathrm{~mm}$. extending between the pulmonary artery and the aorta which it joins just beyond the level of the innominate artery. The foramen ovale has a narrow crescentic opening along the lower border, fairly well covered by the limbus. The interventricular septum is entirely closed.

Lungs: Were removed with the heart. They are of a pale, grayish red color, give crepitation and on section the bronchi show some yellowish, frothy fluid escaping. The trachea and bronchi are normal.

Spleen: Weight, $15 \mathrm{gm}$. It is very dark red and the surface is tense. The pulp is markedly congested. There are two accessory spleens close to the hilum, measuring 2 and $3 \mathrm{~mm}$. in size, respectively.

Gastro-Intestinal Tract: Shows considerable edema and congestion throughout.

Liver: Weighs $98 \mathrm{gm}$. and is markedly congested.

Kidneys: Weigh $12 \mathrm{gm}$. each. The fetal lobulations are still conspicuous. The cortex and medulla are moderately congested.

Aorta: There is no abnormality in the size or branching.

\section{DISCUSSION}

The developmental errors which result in complete transposition of the aorta and pulmonary artery are chiefly two, according to Rokitansky, ${ }^{1}$ who gave the first reasonable explanation for the phenomenon. These are: (a) a deviation of the aortic septum within the truncus aorticus, and (b) its faulty union with the interventricular septum.

Early in fetal life the elongated truncus aorticus is divided into aorta and pulmonary artery by a process in which three factors each play a part. These are, first, a proximal and, second, a distal part of the endocardial swellings in the bulbar part of the truncus aorticus which are known as the proximal and distal bulbar swellings and, third, the aortopulmonary septum. These three elements, at first distinct, later blend to form the spiral septum with its concavity facing posteriorly and to the right, dividing the truncus aorticus into aorta and pulmonary artery. With the ventricles in their normal position any deviation of the septum will cause a change in the relative positions of

1. Rokitansky: Defekte der Scheidewande des Herzens, Vienna, 1875. 
the two vessels and transposition will result, the aorta arising from the right ventricle and the pulmonary artery from the left ventricle.

The part played by faulty union of the aortic septum with the interventricular septum may be very important. Quoting from Abbott ${ }^{2}$ ". . . in the normal heart, in the union of the aortic and interventricular septa, the latter becomes continuous with the left wall of the aorta in front and with its right wall behind (through the pars membranacea), surrounding the vessel on its right side in such a way that the latter becomes placed in the left ventricle, while the pulmonary artery comes to lie in the right. Should this union take place at an abnormal angle, so that the interventricular septum surrounds the vessels rising posteriorly, on its left instead of its right wall, the trunks will be placed in the reversed cavities and again, even though the trunks may be in a normal relation to each other, transposition will ensue."

Complete transposition is incompatible with long life. Emanuel's patient, $^{3}$ who lived eleven years, is the oldest case on record; most patients varied in age from a few days to several months. The maintaining of the pulmonary circulation is of greatest importance, and some means must be provided for supplying oxygenated blood to the aorta. The fetus requires no special mechanism, as the pulmonary circulation has not been established. If in an infant with complete transposition immediately after birth the foramen ovale and ductus arteriosus become closed as normally occurs, then the aorta would be supplied with venous blood from the right ventricle and the pulmonary circulation through the left side of the heart and lungs would be a closed circuit. However, cardiac anomalies are seldom single, particularly in the major errors and some means which, nevertheless, is almost always insufficient is provided for passage of oxygenated blood from the pulmonary system to the aorta.

The usual accessories are a patent foramen ovale, a patent ductus arteriosus, a perforate interventricular septum or combination of these. With a widely open foramen ovale blood from the lungs can pass back into the right auricle to escape into the aorta through the right ventricle. Case 1 had such a channel and lived a year even with a patent interventricular septum closed by granulations. An open ductus arteriosus is probably seldom of sufficient caliber to. compensate as well as a fully open foramen ovale and Case 2 with a very slight aperture for a foramen and a patent ductus with a caliber of about $3 \mathrm{~mm}$. was insufficient to supply oxygenated blood to the systemic arterial circulation, the patient living but six weeks.

2. Abbott: Osler's Modern Medicine 4:368.

3. Emanuel: Reference in Abbott's Article, Osler's Modern Medicine 4:368. 
In Case 1 there was also an acquired tricuspid insufficiency. With this additional lesion the mechanics of the circulation might be explained as follows: During right auricular systole, blood would be propelled both through the tricuspid valve and through the foramen ovale. However, at systole of the left auricle there would tend to be a current through the foramen from left to right. This blood would be oxygenated and be mixed with the venous blood of the right auricle but likely not in equal proportions on account of the muscular preponderance of the right side of the heart and the regurgitation through the tricuspid valve. These two factors would produce a pressure in the right auricle greater than that in the left and a mixture of blood from the two sides made difficult. Consequently, there would tend to be an anoxemia in the systemic circulation and it is thus seen that the tricuspid insufficiency undoubtedly was an important contributory factor in the death of the patient.

In Case 2 there was a foramen ovale of very small proportions, too small to give any symptoms in an otherwise normal heart. The ductus arteriosus was open but its lumen was too small to compensate for the abnormal origin of the great vessels. The life of the child was consequently of short duration, six weeks, in striking contrast to the first child with a widely patent foramen ovale and who lived a year. 